\title{
Ab Initio Study of Electronic Transport in Cubic-HfO Grain Boundaries
}

\author{
Elena Degoli, ${ }^{1,2}$ Eleonora Luppi, ${ }^{3,4}$ and Nathalie Capron ${ }^{5,6}$ \\ ${ }^{1}$ Dipartimento di Scienze e Metodi dell'Ingegneria, Università di Modena e Reggio Emilia, Via Amendola 2 Padiglione Morselli, \\ 42122 Reggio Emilia, Italy \\ ${ }^{2}$ CNR-Istituto di Nanoscienze-S3, Via Campi 213/A, 41125 Modena, Italy \\ ${ }^{3}$ Laboratoire de Chimie Théorique, Sorbonne Universités, UPMC Univ Paris 06, UMR 7616, 75005 Paris, France \\ ${ }^{4}$ CNRS, UMR 7616, Laboratoire de Chimie Théorique, 75005 Paris, France \\ ${ }^{5}$ Laboratoire de Chimie Physique-Matière et Rayonnement, Sorbonne Universités, UPMC Univ Paris 06, UMR 7614, \\ 75005 Paris, France \\ ${ }^{6}$ CNRS, UMR 7614, Laboratoire de Chimie Physique-Matière et Rayonnement, 75005 Paris, France
}

Correspondence should be addressed to Elena Degoli; elena.degoli@unimore.it

Received 18 March 2017; Accepted 25 May 2017; Published 5 July 2017

Academic Editor: Mohamed Bououdina

Copyright (c) 2017 Elena Degoli et al. This is an open access article distributed under the Creative Commons Attribution License, which permits unrestricted use, distribution, and reproduction in any medium, provided the original work is properly cited.

\begin{abstract}
In polycrystalline materials the grain boundaries (GBs) are particularly important as they can act as a sink for atom defects and impurities, which may drive structural transformation of the materials and consequently modify their properties. Characterising the structure and properties of GBs is critical for understanding and controlling material property. Here, we investigated how GBs can modify the structural, electronic, and transport properties of the polycrystalline material $\mathrm{HfO}_{2}$. In general, grain boundaries are considered to be detrimental to the physical stability and electronic transport in $\mathrm{HfO}_{2}$. Anyway, studying by first principles the two most stable and common types of GBs, the tilt and the twist, we found substantial differences on the impact they have on the material properties. In fact, while tilt defects create channels of different sizes and shapes in hafnia along which the electronic transport is stronger in relation to leakage current through GBs, twist defects create a sort of amorphous structure that tends to resemble the bulk and which is independent of the number of rotated planes/atoms.
\end{abstract}

\section{Introduction}

Grain boundary (GB) in a solid crystalline material is a region separating two crystals (grains) of the same phase. These two grains differ in mutual orientations and the grain boundary represents a transition region, where the atoms are shifted from their regular positions as compared to the crystal. In general, polycrystalline materials contain a wide variety of interfaces between grains that can be influenced by numerous factors, such as growth conditions and thermal treatment.

In polycrystalline materials the GB is particularly important as it can act as a sink for atom defects and impurities, which may drive structural transformation of the materials and consequently modify their properties. In fact, GBs can change the mechanical strength, thermal dissipation, and electrical transport of the crystals and have consequences on their performance for specific applications in optoelectronics and photovoltaics. Therefore, characterising the structure and properties of grains and grain boundaries is critical for understanding and controlling material property.

GBs appeared to severely weaken the mechanical strength [1] and to impede electrical transport [2] and transmission [3] of graphene. In crystalline monolayer molybdenum disulphide $\mathrm{MoS}_{2}$, depending on the type of GB formed in the crystal, an enhancement or a quenching of the photoluminescence and electrical conductivity was observed [4]. The presence of GBs can also trap electrons in semiconducting and insulating materials which is important for optoelectronic and photovoltaics applications [5]. Moreover, recently the presence of GBs in Si has been studied in order to engineer 
these defects to improve electronic and optical properties of $\mathrm{Si}$ in photovoltaic devices [6].

In recent years, a particular interest was given to understand the role of GBs in the modification of the electronic and transport properties of transition-metal oxide hafnia $\left(\mathrm{HfO}_{2}\right)$. In fact, $\mathrm{HfO}_{2}$ is nowadays considered the most promising candidate to replace silicon dioxide $\left(\mathrm{SiO}_{2}\right)$ as the gate dielectric material in metal-oxide-semiconductors (MOS) and complementary MOS (CMOS) technology [7, 8]. Moreover, $\mathrm{HfO}_{2}$ is also very promising as material for resistive random access memories (RRAMs) $[9,10] . \mathrm{HfO}_{2}$ has wide bandgap $(5.7 \mathrm{eV})$ and high dielectric constant (22) and exhibits good thermodynamic stability. However, $\mathrm{HfO}_{2}$ suffers from important current leakage which has been related to neutral and charged oxygen vacancies $[11,12]$ and oxygen interstitials $[13,14]$ close to region where the GB is formed in $\mathrm{HfO}_{2}$. The leakage currents are related to the structure of the GB which presents favourable percolation paths for electron tunnelling [15-17]. For this reason, GBs are generally considered to be detrimental to the physical stability and electronic transport in $\mathrm{HfO}_{2}$. A clearer understanding and controlling of GBs become essential for optimising device performance and reliability.

The aim of this work is to investigate how the GBs can modify the structural, electronic, and transport properties of the polycrystalline material $\mathrm{HfO}_{2}$. We decided to start considering $\mathrm{HfO}_{2}$ in its cubic phase and to construct two different types of GBs: tilt and twist. We first studied the changes in the structural and electronic properties of cubic $\mathrm{HfO}_{2}$ due to the presence of GBs. Next, we investigated the transport properties in the framework of the Boltzmann transport equations. In order to interpret the results with respect to the experimental value we interpret the oxygen vacancy as donor which means that the system is n-doped and the interstitial oxygen as acceptor which means that the system is p-doped [18]. We do not consider possible relaxation or change in the band structures. The change of the doping is discussed as a function of the chemical potential. With this approach we want to clarify the mechanisms of electronic transport in GBs. We expect that our findings are general enough to be relevant in experimental conditions.

\section{Computational Methodology}

The structural and electronic properties of the systems studied in this work were calculated using first-principle density functional theory (DFT) as implemented in the plane-wave based Vienna Ab initio Simulation Package (VASP) $[19,20]$. We employed the generalised gradient approximation (GGA) for the exchange-correlation functional in the parametrisation proposed by Perdew and Wang (PW91) [21]. We adopted projector augmented-wave pseudopotentials including the valence $5 p, 5 d$, and 6 s electrons for $\mathrm{Hf}$ and the $2 \mathrm{~s}$ and $2 \mathrm{p}$ electrons for $\mathrm{O}$. We used a plane-wave cut-off of $400 \mathrm{eV}$ and $\Gamma$ centered Monkhorst-Pack [22] $k$-mesh of $9 \times 9 \times$ 9 for the bulk, of $24 \times 8 \times 12$ for the tilt, and of $12 \times$ $12 \times 16$ for the twist GB structures. All the structures were relaxed until the Hellmann-Feynman forces exerted on each atom become less than $0.01 \mathrm{eV} / \AA$. The electronic

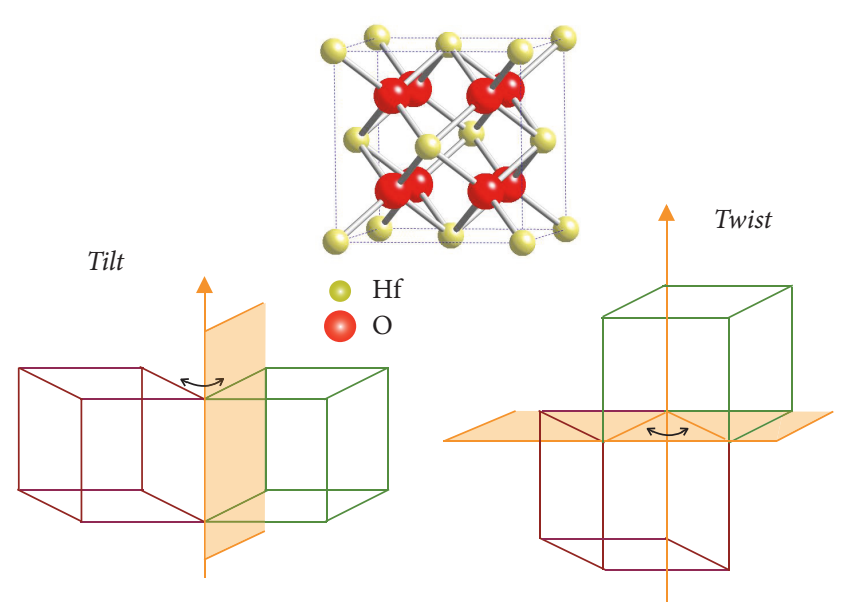

FIgURE 1: The cubic phase of $\mathrm{HfO}_{2}$ is presented where $\mathrm{Hf}$ atoms are in yellow and $\mathrm{O}$ atoms in red. A cartoon representation is presented to schematise the way tilt (left) and twist (right) GBs are constructed.

transport properties were calculated using the BoltzTraP (BoltzmannTransportProperties) code [23]. In particular, we analysed the conductivity $\sigma$ in the rigid-band and constant relaxation-time approximation.

\section{Results}

3.1. Theoretical Models for $\mathrm{HfO}_{2}$ Grain Boundaries. We started by considering $\mathrm{HfO}_{2}$ in its cubic phase and we found a lattice parameter of $5.07 \AA$, in agreement with other theoretical calculations and experiments [24-27]. From the cubic $\mathrm{HfO}_{2}$ we obtained tilt GBs by cutting the crystal along the $(0-13)$ plane and rotating it of $36.86^{\circ}$ with respect to the $\left[\begin{array}{lll}1 & 0 & 0\end{array}\right]$ axis belonging to the plane of the boundary (see left panel of Figure 1). In a similar way we built the twist GBs by cutting the cubic $\mathrm{HfO}_{2}$ along the ( $\left.\begin{array}{lll}3 & 1 & 0\end{array}\right)$ plane and by rotating it of $36.86^{\circ}$ with respect to the $\left[\begin{array}{ll}0 & 0\end{array}\right]$ axis perpendicular to the plane of the boundary (see right panel of Figure 1).

In this way, we constructed two tilt GBs of the type $\Sigma 5$ $36.86^{\circ}\left(\begin{array}{lll}0 & -1 & 3\end{array}\right) /\left[\begin{array}{lll}1 & 0 & 0\end{array}\right]$ and two twist GBs of the type $\Sigma 5$ $36.86^{\circ}\left(\begin{array}{lll}3 & 1 & 0\end{array}\right) /\left[\begin{array}{lll}0 & 0 & 1\end{array}\right]$. The $\Sigma 5$ notation refers to the degree of site matching. $\Sigma 5$ indicates a high symmetry and possibly low formation energy. $\Sigma$ is simply evaluated as

$$
\Sigma=\delta \times\left(h^{2}+k^{2}+l^{2}\right),
$$

where $(h k l)$ is the plane of the boundary and $\delta$ is equal to 1 as $\left(h^{2}+k^{2}+l^{2}\right)$ is odd in our case [25]. The two tilt GBs differ in the number of atoms per unit cell: 72 atoms (Tilt72) and 108 atoms (Tilt108), and they are shown in Figure 2 before and after geometrical relaxation. The unit cell is orthorhombic and in the case of Tilt72 the cell parameters are $a=5.11 \AA$, $b=16.16 \AA$, and $c=9.70 \AA$ while in the case of the Tilt108 the parameters are $a=5.11 \AA, b=16.16 \AA$, and $c=14.54 \AA$. In the case of the twist GBs the number of atoms per unit cell is 90 (Twist90) and 120 (Twist120) and they are shown in Figure 3 before and after geometrical relaxation. The unit cell is tetragonal and for the Twist90 the cell parameters are 

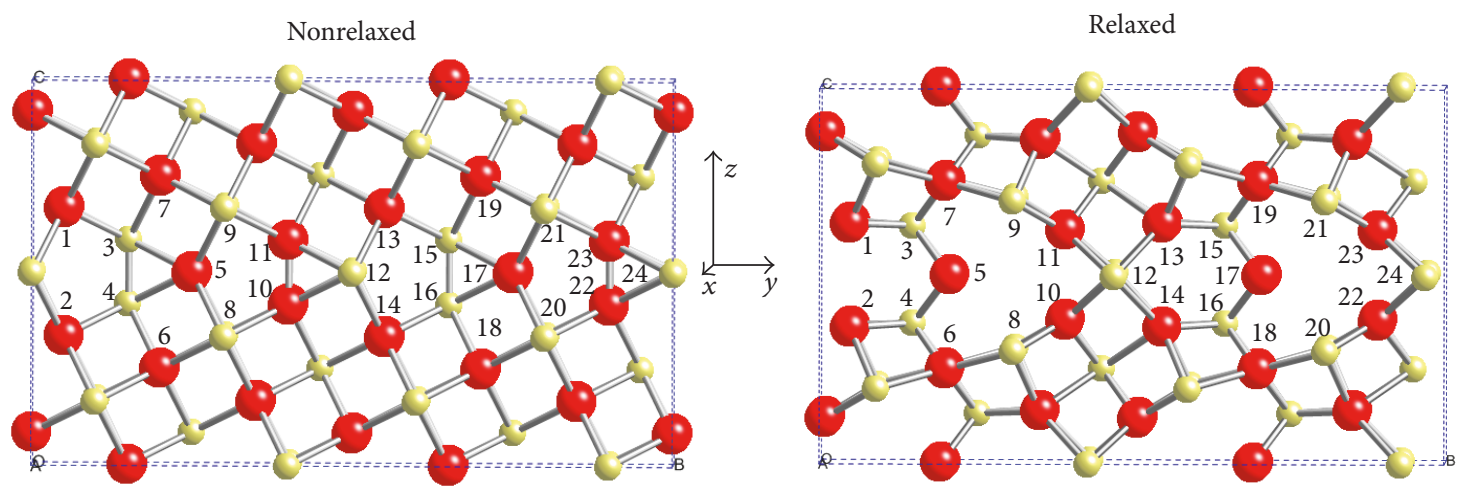

Tilt108
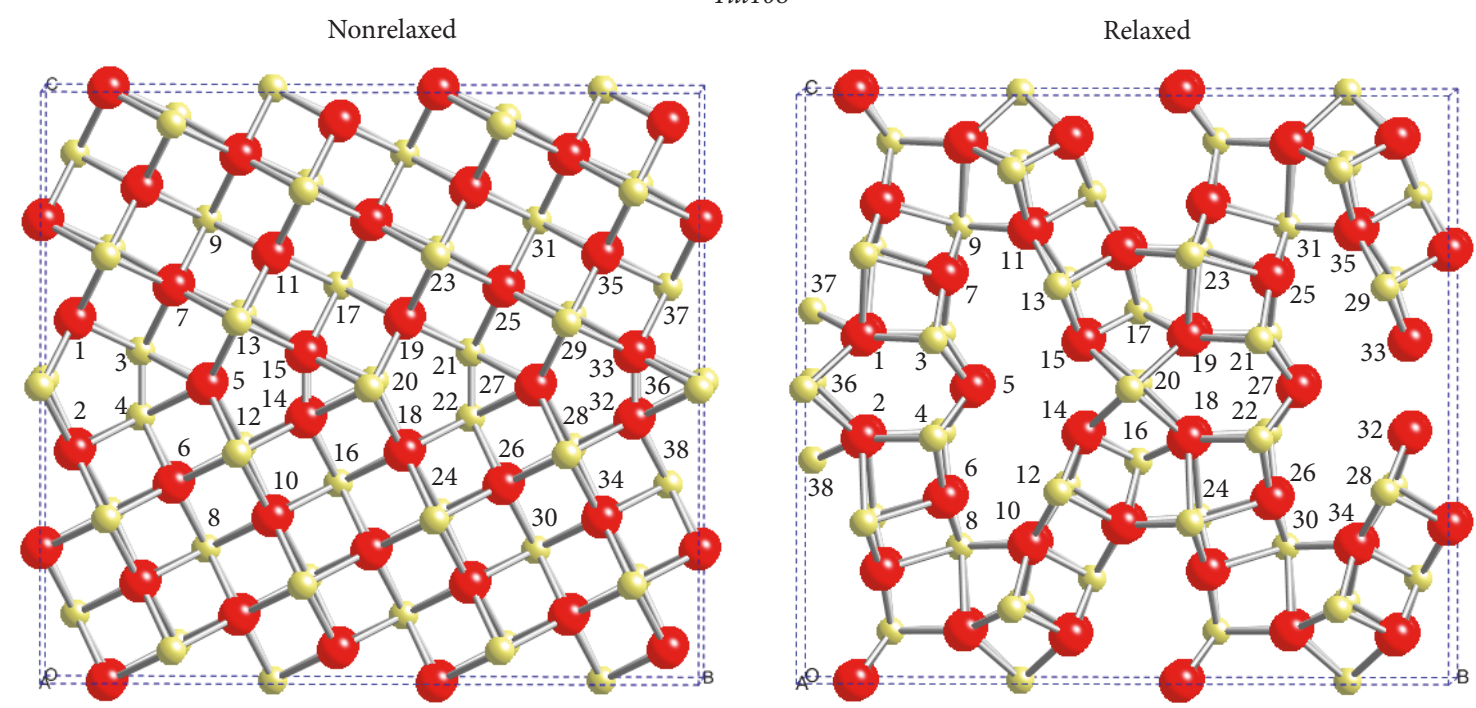

Figure 2: Tilt72 and Tilt108 are shown before and after geometrical relaxation.

$a=b=5.11 \AA$ and $c=8.18 \AA$ while for the Twist120 they are $a=b=5.11 \AA$ and $c=10.22 \AA$.

The effects of the geometrical relaxation in the GBs structures are immediately observable in Figures 2 and 3 and quantitatively shown in Tables 1 and 2 where we reported the distances between the atoms before $\left(d_{n R}\right)$ and after relaxation $\left(d_{R}\right)$, together with the percentage of relaxation $(R)$. This percentage was calculated as $R=\left(d_{R}-d_{n R}\right) / d_{n R} * 100$.

In the case of the Tilt72, we labelled from 1 to 24 the atoms which play the most important role in the relaxation. All these atoms are symmetric with respect to the $x y$ plane. The geometrical relaxation creates two almost spherical large channels and two small channels with a hexagonal structure. The initial distances between first neighbouring atoms are of $2.213 \AA$ and then there is a compensation between elongation and compression which is at the origin of these channels. In particular, we observe that the bigger channels are due to a strong modification of the distances between atoms 5 (17) and 9 (21) and between atoms 5 (17) and 8 (20). The percentage of deformation is $R=29.9 \%$. The deformation can be also investigated by studying the change of distances along the $z$ direction. Here, the maximum deformation is related to atoms 3 (15) and 4 (16) which is $R=56.4 \%$. Those atoms that do not play a role in the creation of the channels still keep the initial bulk distances. As a consequence, when repeating the unit cell in the space, we found that our structures are made of small and large channels with bulk-like regions in between. The mean extension of these regions is of the order of $6 \AA$.

A similar result was obtained for the Tilt108 where we labelled from 1 to 38 the atoms which play the most important role in the relaxation. Also in this case, all the atoms are symmetric with respect to the plane of symmetry $x O y$ before and after the geometrical optimisation. The relaxation creates two elongated large and small channels still with a hexagonal form. The smaller channels have similar relaxation distances and percentages of the Tilt72. The elongated channels are due to a strong modification of the distances between atoms 5 (27) and 13 (29) and between atoms 5 (27) and 12 (28). The percentage of deformation is $R=62.7 \%$. Also in this case, those atoms that do not play a role in the creation of the 
TABLE 1: We report the distances between the atoms as labelled in Figure 2 for Tilt72 and Tilt108 before $\left(d_{n R}\right)$ and after geometry relaxation $\left(d_{R}\right)$. We also report the percentage of relaxation $R$ which is calculated as $R=\left(d_{R}-d_{n R}\right) / d_{n R} * 100$.

\begin{tabular}{|c|c|c|c|}
\hline & $d_{n R}(\AA)$ & $d_{R}(\AA)$ & $R(\%)$ \\
\hline \multicolumn{4}{|c|}{ Tilt72 } \\
\hline$d_{1-3}=d_{2-4}=d_{13-15}=d_{14-16}$ & 2.213 & 2.070 & -6.46 \\
\hline$d_{3-5}=d_{4-5}=d_{15-17}=d_{16-17}$ & 2.213 & 2.016 & -8.90 \\
\hline$d_{3-7}=d_{4-6}=d_{15-19}=d_{16-18}$ & 2.213 & 1.979 & -10.57 \\
\hline$d_{5-9}=d_{5-8}=d_{17-21}=d_{17-20}$ & 2.213 & 2.875 & 29.9 \\
\hline$d_{7-9}=d_{6-8}=d_{19-21}=d_{18-20}$ & 2.213 & 2.190 & -1.04 \\
\hline$d_{9-11}=d_{8-10}=d_{21-23}=d_{20-22}$ & 2.213 & 1.971 & -10.94 \\
\hline$d_{11-12}=d_{10-12}=d_{23-24}=d_{22-24}$ & 2.213 & 2.114 & -4.47 \\
\hline$d_{12-13}=d_{12-14}=d_{24-1}=d_{24-2}$ & 2.213 & 2.231 & 0.81 \\
\hline$d_{1-2}=d_{13-14}$ & 3.232 & 2.685 & -16.9 \\
\hline$d_{3-4}=d_{15-16}$ & 1.616 & 2.527 & 56.4 \\
\hline$d_{7-6}=d_{19-18}$ & 4.848 & 4.848 & 0.0 \\
\hline$d_{9-8}=d_{21-20}$ & 3.232 & 3.895 & 20.5 \\
\hline$d_{11-10}=d_{23-22}$ & 1.616 & 2.325 & 43.9 \\
\hline$d_{5-12}=d_{17-24}$ & 4.237 & 4.453 & 5.1 \\
\hline$d_{12-17}=d_{24-5}$ & 4.237 & 4.037 & -4.7 \\
\hline \multicolumn{4}{|c|}{ Tilt108 } \\
\hline$d_{1-3}=d_{2-4}=d_{19-21}=d_{18-22}$ & 2.213 & 2.179 & -1.5 \\
\hline$d_{3-5}=d_{4-5}=d_{21-27}=d_{22-27}$ & 2.213 & 1.966 & -1.1 \\
\hline$d_{3-7}=d_{4-6}=d_{21-25}=d_{22-26}$ & 2.213 & 1.930 & -1.27 \\
\hline$d_{5-13}=d_{5-12}=d_{27-29}=d_{27-28}$ & 2.213 & 3.602 & 62.7 \\
\hline$d_{7-9}=d_{6-8}=d_{25-31}=d_{26-30}$ & 2.213 & 1.880 & -15.0 \\
\hline$d_{7-13}=d_{6-12}=d_{25-29}=d_{26-28}$ & 2.213 & 3.114 & 40.7 \\
\hline$d_{9-11}=d_{8-10}=d_{31-35}=d_{30-34}$ & 2.213 & 2.104 & -4.9 \\
\hline$d_{11-13}=d_{10-12}=d_{29-35}=d_{28-34}$ & 2.213 & 2.081 & -5.9 \\
\hline$d_{13-15}=d_{12-14}=d_{29-33}=d_{28-32}$ & 2.213 & 1.953 & -11.7 \\
\hline$d_{15-20}=d_{14-20}=d_{33-36}=d_{32-36}$ & 2.213 & 2.116 & -4.4 \\
\hline$d_{20-19}=d_{20-18}=d_{36-1}=d_{36-2}$ & 2.213 & 2.192 & -1.0 \\
\hline$d_{5-15}=d_{5-14}=d_{27-33}=d_{27-32}$ & 2.213 & 2.971 & 34.2 \\
\hline$d_{1-2}=d_{19-18}$ & 3.232 & 2.524 & -21.9 \\
\hline$d_{3-4}=d_{21-22}$ & 1.616 & 2.439 & 50.9 \\
\hline$d_{7-6}=d_{25-26}$ & 4.848 & 5.489 & 13.2 \\
\hline$d_{9-8}=d_{31-30}$ & 8.080 & 7.921 & -2.0 \\
\hline$d_{11-10}=d_{35-34}$ & 6.464 & 7.771 & 20.2 \\
\hline$d_{13-12}=d_{29-28}$ & 3.232 & 5.065 & 56.7 \\
\hline$d_{15-14}=d_{33-32}$ & 1.616 & 2.288 & 56.4 \\
\hline$d_{17-16}=d_{37-38}$ & 4.848 & 3.709 & 23.5 \\
\hline$d_{5-20}$ & 4.237 & 4.188 & -1.2 \\
\hline$d_{20-27}$ & 4.237 & 4.285 & -1.1 \\
\hline
\end{tabular}

channels still keep the initial bulk distances leaving bulk-like regions in between channels. The mean extension of these regions is of the order of $6.3 \AA$.

The twist GBs have a quite different behaviour. The rotation with respect to an axis perpendicular to the plane of the grain boundary does not create any channels in the structures as shown in Figure 3 for the Twist90 and Twist120. In fact, the twist GB alternates planes of $\mathrm{O}$ and $\mathrm{Hf}$ with a different inclination. Through an analysis of the $\mathrm{O}$ and Hf distances, before and after relaxation, we observed that the structure has a general tendency to rearrange as in the bulk even if it is more compact. After relaxation, the Hf-O distances have almost the same value which is around $2.000 \AA$ which is close but smaller than the one calculated in the bulk of $2.195 \AA$. We showed this behaviour in Table 2 where we considered an Hf atom at the interface between the twisted planes and its distances with the neighbouring $\mathrm{O}$ atoms.

Moreover, it is also worth noticing the effect imposed by the size of the periodic cell on the structural relaxation of tilt and twist GBs. In the case of the tilt, going from 72 to 108 

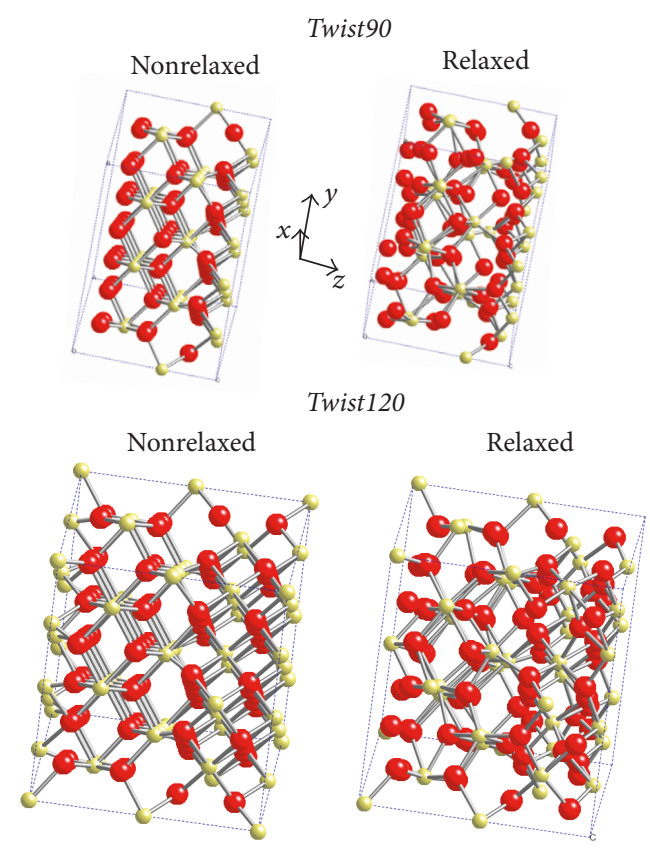

Figure 3: Twist 90 and Twist120 are shown before and after geometrical relaxation.

TABLE 2: Distances between Hf at the interface of the twisted planes and its neighbouring $\mathrm{O}$ atoms for Twin 90 and Twin120 before and after relaxation. We also report the percentage of relaxation $R$ which is calculated as $R=\left(d_{R}-d_{n R}\right) / d_{n R} * 100$. In the bulk the distance between $\mathrm{O}$ and $\mathrm{Hf}$ is $2.195 \AA$.

\begin{tabular}{lccc}
\hline & $d_{n R}(\AA)$ & $d_{R}(\AA)$ & $R(\%)$ \\
\hline \multicolumn{3}{c}{ Twin90 } & \\
$d_{1}$ & 1.512 & 2.064 & 36.51 \\
$d_{2}$ & 2.213 & 2.000 & -9.62 \\
$d_{3}$ & 2.213 & 2.006 & -9.35 \\
$d_{4}$ & 2.213 & 2.032 & -8.18 \\
$d_{5}$ & 2.213 & 2.170 & -1.94 \\
$d_{6}$ & 2.213 & 2.199 & -0.63 \\
\hline & & Twin120 & \\
$d_{1}$ & 1.512 & 2.017 & 33.40 \\
$d_{2}$ & 2.213 & 1.988 & -10.17 \\
$d_{3}$ & 2.213 & 1.996 & -9.81 \\
$d_{4}$ & 2.213 & 2.074 & -6.70 \\
$d_{5}$ & 2.213 & 2.113 & -4.82 \\
$d_{6}$ & 2.213 & 2.160 & -2.56 \\
\hline
\end{tabular}

atoms imposes a stronger constraint on the structure which creates larger channels. Instead, in the case of the twist, going from 90 to 120 atoms does not really seem to change. In both cases the structure tries to rearrange as in the bulk.

3.2. Formation Energies. The stability of the GBs was studied by calculating the formation energy of the grain boundary with respect to the cubic $\mathrm{HfO}_{2}$. In Table 3 we indicated for each structure, in parenthesis, the number of motifs $\left(n_{\text {motif }}\right)$ per unit cell. We also reported the total energy and the total
TABLE 3: For each structure studied, we report the number of motif, $n_{\text {motif }}$, in the unit cell (in parenthesis), the total energies $E(\mathrm{eV})$, the total energies per motif $E / n_{\text {motif }}(\mathrm{eV})$ and the formation energies $E_{f}$ $(\mathrm{eV})$ which are calculated as $E_{f}=\left(E_{\mathrm{GB}} / n_{\text {motif }}-E_{\text {bulk }} / n_{\text {motif }}\right)$.

\begin{tabular}{lcccc}
\hline & $E(\mathrm{eV})$ & $E / n_{\text {motif }}(\mathrm{eV})$ & $E_{f}(\mathrm{eV})$ & $E_{f}\left(\mathrm{~J} / \mathrm{m}^{2}\right)$ \\
\hline Bulk (4) & -121.49 & -30.37 & & \\
Twist90 (30) & -896.83 & -29.89 & 0.48 & 0.29 \\
Twist120 (40) & -1202.51 & -30.06 & 0.31 & 0.19 \\
Tilt72 (24) & -691.62 & -28.82 & 1.55 & 0.30 \\
Tilt108 (36) & -1011.99 & -28.11 & 2.26 & 0.43 \\
\hline
\end{tabular}

energy per motif to calculate the formation energy of the GB as

$$
E_{f}=\left(\frac{E_{\mathrm{GB}}}{n_{\text {motif }}}-\frac{E_{\text {bulk }}}{n_{\text {motif }}}\right) .
$$

$E_{f}$ of the twist GBs is lower than tilt GBs which demonstrates that twist GBs are the most stable structures. We put these evidences in relation to the important structural differences between tilt and twist GB. Even, if the twist structures loose most of the symmetries of the $\mathrm{HfO}_{2}$ in the bulk phase, the distances between the atoms are very close to those of the cubic $\mathrm{HfO}_{2}$, while in the case of the tilt structures the creation of channels induces an extensive rearrangement of the atoms at the interface as confirmed from the calculated distances reported in Table 1. Therefore, the tilt and twist GBs induce a substantially different redistribution of density charge in the structures which is manifest in the very different formation energies. Moreover, differences exist within the same class of GBs. Actually, while for the twist GBs $E_{f}$ is very similar for both structures, the tilt GBs are very sensitive to their dimension: the larger the tilt GBs are, the larger its formation energy is (i.e., the system is progressively less stable).

In literature, tilt GBs have been studied which have a different formation energy per unit area. McKenna et al. [15, 17] investigated a monoclinic $\mathrm{HfO}_{2}$ tilt $\mathrm{GB}$ with a formation energy of $0.6 \mathrm{~J} / \mathrm{m}^{2}$. This has a relatively low formation energy with respect to others they studied and it was chosen based on the principle that the most stable boundaries should have a high degree of site coincidence between grains. Instead, Xue et al. [25] studied cubic $\mathrm{HfO}_{2}$ and reported higher formation energy of $2.92 \mathrm{~J} / \mathrm{m}^{2}, 3.14 \mathrm{~J} / \mathrm{m}^{2}$, and $3.50 \mathrm{~J} / \mathrm{m}^{2}$.

3.3. Electronic Properties. We investigated also which is the effect of the different atoms rearrangements and charge redistributions of tilt and twist GBs on their electronic properties. We always consider as our reference the cubic$\mathrm{HfO}_{2}$ bulk for which we obtained an energy band gap $\left(E_{g}\right)$ of $3.81 \mathrm{eV}$ (in good agreement with literature [28]). The energy gap for the GBs studied diminished with respect to the bulk and this is particularly evident for the tilt defects. In fact, we obtain $E_{g}=0.84 \mathrm{eV}$ for the Tilt72, $E_{g}=0.27 \mathrm{eV}$ for the Tilt108, $E_{g}=2.84 \mathrm{eV}$ for the Twist90, and $E_{g}=3.31 \mathrm{eV}$ for the Twist120.

In Figure 4 we also report the density of states (DOS) where, for all the systems, we took the zero energy reference 


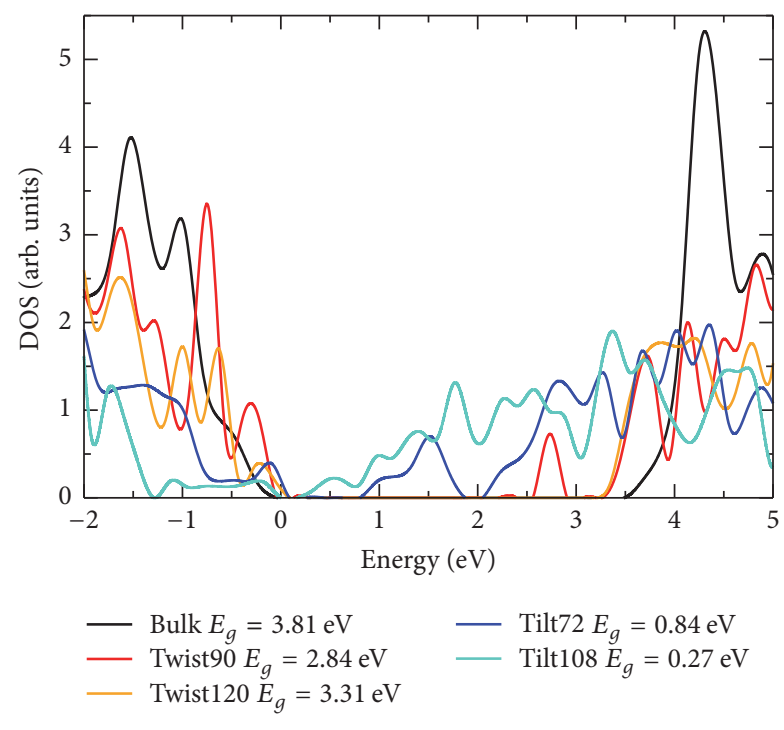

Figure 4: DOS and energy gaps of the tilt and twist GBs studied.

to be the valence band maximum energy. The DOS is reported in an energy range close to the bulk gap energy region as our goal is to investigate the presence of new states within the gap. In fact, the current leakage measured in GBs can be due to electron trapping and tunnelling in the band gap [15, 17].

The Twist90 and Twist120 have few new states close to the conduction bands that slightly diminish the cubic $\mathrm{HfO}_{2}$ gap. Instead, the tilt GBs present many new localised states that reduce the gaps of around $2 \mathrm{eV}$. These new states are almost flat and are due to the channels formation in the bulk structure. These new states induce a transition in $\mathrm{HfO}_{2}$ from insulator to semiconductor.

From these observations, we conclude that tilt GB defect seems to be favourite for current leakage with respect to twist GBs. However, tilt defect seems to be the most advantageous to tune the electronic properties of $\mathrm{HfO}_{2}$ as it permits a higher degree of manipulation.

The presence of these new states in the gap has been observed also in the GBs presented in $[15,17,25]$. However, it is worth noticing that the type of modulation of these new states in the gap strongly depends on the type of GBs. In fact, this defect can create a different number of undercoordinated $\mathrm{O}$ and $\mathrm{Hf}$ at the origin of these states.

To deepen the origin of these new states inside the gap, we plotted in Figure 5 the isosurfaces of the band-decomposed partial charge density considering for the valence the energy range, $\mathrm{HOMO}-1 \mathrm{eV}(\mathrm{a})$, and for the conduction the energy range: $\mathrm{LUMO}+1 \mathrm{eV}$ (b). For both tilt and twist GBs the valence states are localised on the oxygen atoms while the states in the conduction are localised on the Hf-O bonds around the Hf atoms. In particular for the tilt, the atoms related to these states seem to be also those which are involved in the channels. The same is observed in the twist but in this case the charge is delocalised on planes related to the rotation of the boundary.
3.4. Boltzmann Electronic Transport: The Conductivity. The correlation between GBs and increased leakage current has been demonstrated both theoretically and experimentally [15-17]. This effect is a result of a number of related factors such as (i) the intrinsic electronic properties of GBs which enhance direct tunnelling relative to the bulk, (ii) segregation of oxygen vacancies which increases trap assisted tunnelling currents, and (iii) ionic transport via interstitial sites [15-17].

Here, we focused on GBs and their intrinsic electronic properties. We interpreted an oxygen vacancy as a donor which means that the system is n-doped and an interstitial oxygen as an acceptor which means that the system is pdoped [18]. Therefore, the GB can be n- and p-doped and the transport properties can therefore be studied as a function of the electron chemical potential in the framework of the Boltzmann transport equations. In particular, we used the Boltzmann transport theory within the constant scatteringtime approximation as implemented in the BoltzTraP code [23].

The Boltzmann equations for transport are obtained in the thermodynamic limit and under some conditions such that the interactions involving more than two particles can be neglected, the collisions are elastic and involve only uncorrelated particles. This means that particles which have already collided are expected not to recollide in the future. This approach is a simplified model to study transport which appeared to work well in many different cases [29-31]. However, this model does not take into account effects such as electron-hole trapping [32].

The Boltzmann equation for the calculation of the electronic conductivity tensor $\sigma_{\alpha \beta}(\mu, T)$ as a function of the chemical potential $\mu$ and temperature $T$ is

$$
\sigma_{\alpha \beta}(\mu, T)=\frac{1}{\Omega} \int \sigma_{\alpha \beta}(\epsilon)\left[-\frac{\partial f_{0}(\mu, T, \epsilon)}{\partial \epsilon}\right] d \epsilon,
$$

where $\alpha, \beta=x, y, z$ are tensor index, $\Omega$ is the volume of the unit cell, $f_{0}$ is the Boltzmann distribution function, and $\sigma_{\alpha \beta}(\epsilon)$ is defined as

$$
\sigma_{\alpha \beta}(\epsilon)=\frac{1}{N} \sum_{i, \mathbf{k}} \sigma_{\alpha \beta}(i, \mathbf{k}) \frac{\delta\left(\epsilon-\epsilon_{i, \mathbf{k}}\right)}{\delta \epsilon} .
$$

$N$ is the number of $k$-points and $\epsilon_{i, \mathbf{k}}$ are the energy bands indexed by bands $(i)$ and $k$-points $(\mathbf{k})$. The quantity $\sigma_{\alpha \beta}(i, \mathbf{k})$ is

$$
\sigma_{\alpha \beta}(i, \mathbf{k})=e^{2} \tau_{i, \mathbf{k}} v_{\alpha}(i, \mathbf{k}) v_{\beta}(i, \mathbf{k}),
$$

where $e$ is the electron charge, $\tau_{i, \mathbf{k}}$ is the relaxation time, and $v_{\alpha}(i, \mathbf{k})=(1 / \hbar)\left(\partial \epsilon_{i, \mathbf{k}} / \partial k_{\alpha}\right)$ component of group velocities.

In our calculations we assume that $\tau_{i, \mathbf{k}}$ is constant. This approximation is often adopted in first-principle calculations $[33,34]$, and despite its simplicity it often turns out to be a good approximation for bulk materials, even in the case of anisotropic systems [35]. Moreover, in our analysis we assume that $\tau$ does not strongly change in GBs with respect to bulk. For this reason, we assume that all the structures have the same value. This is a strong statement, which, however, does not substantially change the results. 
Tilt72

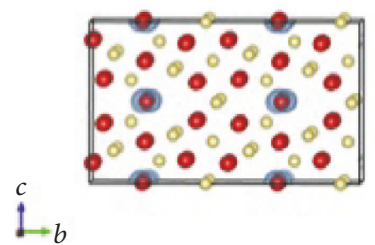

Twist90

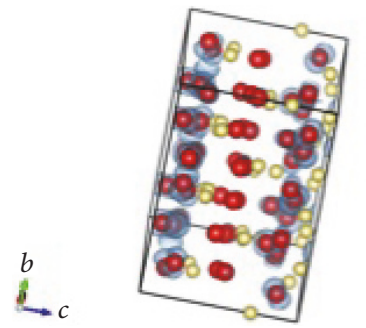

(a)
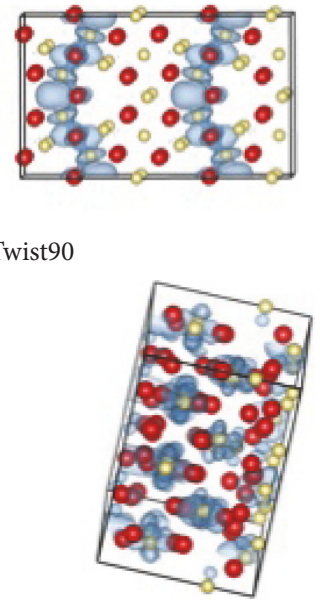

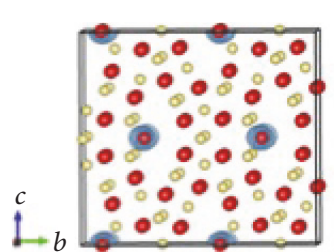

Tilt108

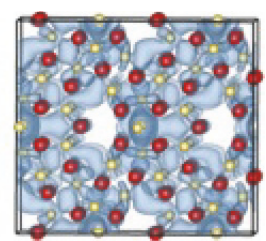

Twist120

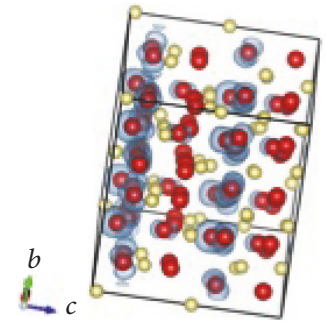

(b)

Figure 5: Isosurfaces of the band-decomposed partial charge density for both tilt and twist GBs. The energy range for the valence states is $\mathrm{HOMO}-1 \mathrm{eV}$ (a) while the energy range for the conduction states is LUMO $+1 \mathrm{eV}(\mathrm{b})$.

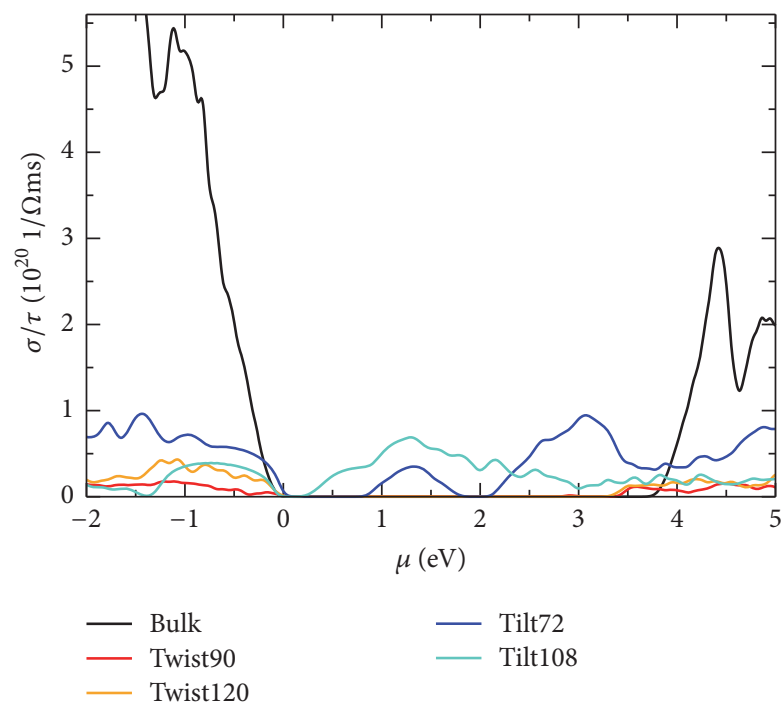

FIGURE 6: $\sigma / \tau$ as a function of the chemical potential for the tilt and twist GBs structures studied.

In Figure 6 we show $\sigma / \tau$ where we used $\tau=1 \mathrm{fs}$ for all the structures [23]. $\sigma / \tau$ as a function of the electron chemical potential has to be interpreted as if the system were $\mathrm{n}$-doped (O vacancies) in the case of positive $\mu$ or $\mathrm{p}$-doped (O interstitials) in the case of negative $\mu$.

Considering the conductivity of bulk as a reference we observe that $\sigma / \tau$ is particularly large for the tilt GBs. The magnitude is higher than twist and it is more important in the gap region. The new electronic states give a strong contribution to increase current.

The behaviour of $\sigma / \tau$ is strongly related to the DOS. The tilt defects create new states in the gap which appeared to be crucial in enhancing electronic transport. Instead, the transport in the twist defects, despite some few new states in the gap, seems to be generally quenched in particular for positive $\mu$.

We also considered the role of anisotropy in electronic transport as we show in Figure 7. In the case of Tilt72 and Tilt108 the anisotropy is much higher than in Twist90 and Twist120. In particular, it is interesting to note that a huge increasing of the conductivity is observed along the direction of the channels in the tilt GBs. These put in evidence the role of the channels in the leakage current.

\section{Conclusions}

We investigated how tilt and twin GBs modify the structural, electronic, and transport properties of the polycrystalline $\mathrm{HfO}_{2}$. The different structural properties of these defects have a strong impact on their electronic and transport properties. In fact, in the case of the tilt it is possible to create channels of different sizes and shapes while for the twist GBs the defect creates a sort of amorphous structure that tends to resemble the bulk, independent of the number of rotated planes/atoms. These structural differences and the strong anisotropy of tilt GBs reflect a major stability of twist with respect to tilt GBs. Nevertheless tilt defects result to be crucial for the enhancing of the electronic transport that results to be stronger along their channels in relation to leakage current through GBs. The different behaviour of the tilt and twist GBs can be used, for example, to properly engineer innovative materials in order to change the electrical resistance of the device between two distinct levels (this is actually a common characteristic of the metal-oxide-based resistance switching memories), that is, the High- and Low-Resistance-State, associated with different atomic arrangements of the same dielectric material.

\section{Conflicts of Interest}

The authors declare that there are no conflicts of interest regarding the publication of this paper. 

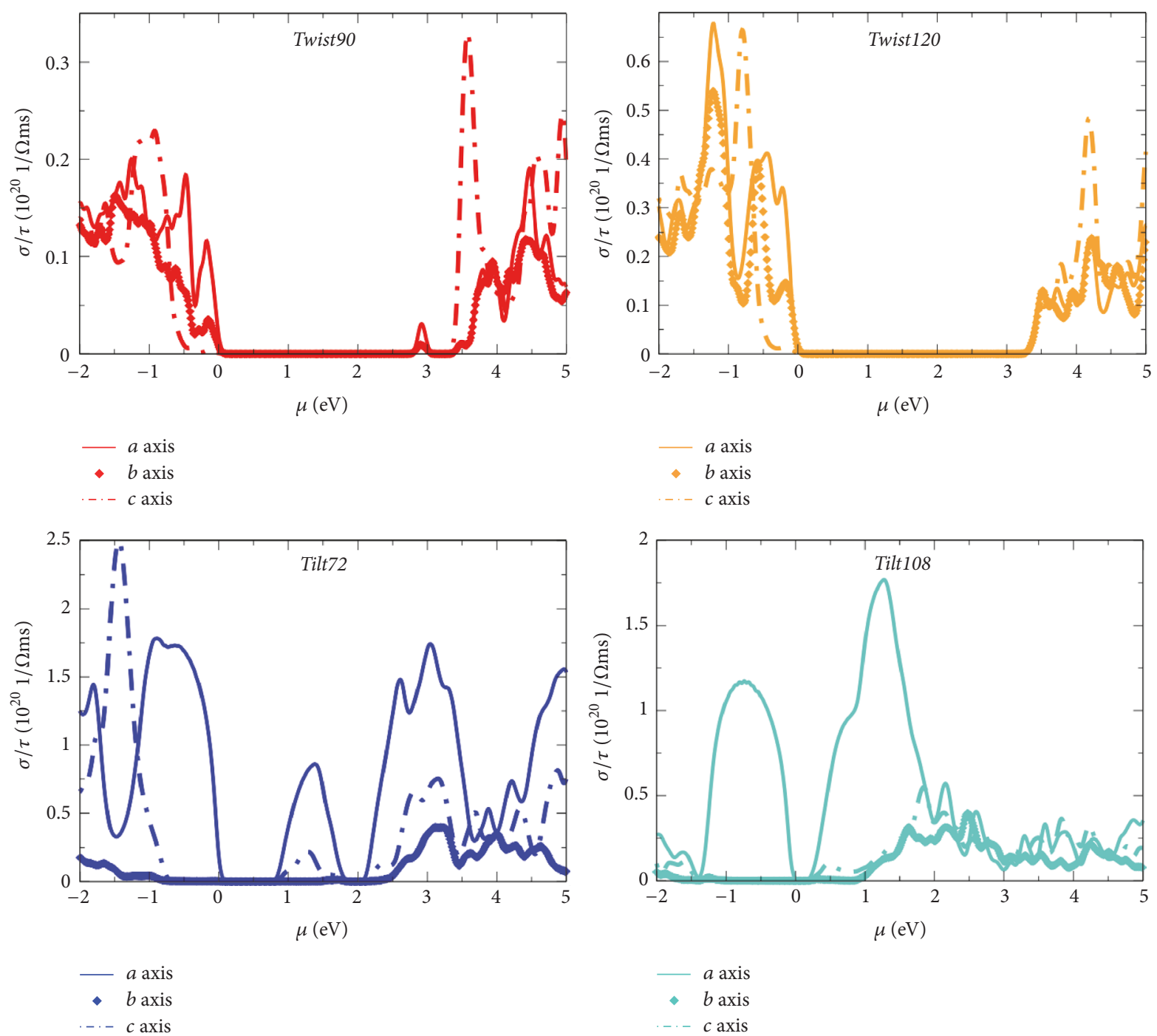

Figure 7: $\sigma / \tau$ projected along $a, b$, and $c$ axis as a function of the chemical potential for the tilt and twist GBs structures studied.

\section{Acknowledgments}

The authors would like to thank Dr. Andrea Ferretti for discussion and acknowledge the (SHO) ISCRA C CINECA project for time machine. They are also grateful to Professor Hafner (Faculty of Physics and Center for Computational Materials Science, University of Vienna, Austria) for allowing them to use the VASP code.

\section{References}

[1] P. Y. Huang, C. S. Ruiz-Vargas, A. M. van der Zande et al., "Grains and grain boundaries in single-layer graphene atomic patchwork quilts," Nature, vol. 469, no. 7330, pp. 389-392, 2011.

[2] Q. Yu, L. A. Jauregui, W. Wu et al., "Control and characterization of individual grains and grain boundaries in graphene grown by chemical vapour deposition," Nature Materials, vol.10, no. 6, pp. 443-449, 2011.

[3] H. Zhang, G. Lee, C. Gong, L. Colombo, and K. Cho, "Grain boundary effect on electrical transport properties of graphene,"
Journal of Physical Chemistry C, vol. 118, no. 5, pp. 2338-2343, 2014.

[4] A. M. Van Der Zande, P. Y. Huang, D. A. Chenet et al., "Grains and grain boundaries in highly crystalline monolayer molybdenum disulphide," Nature Materials, vol. 12, no. 6, pp. 554-561, 2013.

[5] K. P. McKenna and A. L. Shluger, "Electron-trapping polycrystalline materials with negative electron affinity," Nature Materials, vol. 7, no. 11, pp. 859-862, 2008.

[6] R. Raghunathan, E. Johlin, and J. C. Grossman, "Grain boundary engineering for improved thin silicon photovoltaics," Nano Letters, vol. 14, no. 9, pp. 4943-4950, 2014.

[7] B. K. Park, J. Park, M. Cho et al., "Interfacial reaction between chemically vapor-deposited $\mathrm{HfO}_{2}$ thin films and a HF-cleaned Si substrate during film growth and postannealing," Applied Physics Letters, vol. 80, no. 13, pp. 2368-2370, 2002.

[8] E. P. Gusev, C. Cabral Jr., M. Copel, C. D'Emic, and M. Gribelyuk, "Ultrathin $\mathrm{HfO}_{2}$ films grown on silicon by atomic layer deposition for advanced gate dielectrics applications," Microelectronic Engineering, vol. 69, no. 2-4, pp. 145-151, 2003. 
[9] S. Clima, B. Govoreanu, M. Jurczak, and G. Pourtois, "HfOx as RRAM material - First principles insights on the working principles," Microelectronic Engineering, vol. 120, pp. 13-18, 2014.

[10] F. M. Puglisi, P. Pavan, A. Padovani, and L. Larcher, "A study on $\mathrm{HfO}_{2}$ RRAM in HRS based on I-V and RTN analysis," SolidState Electronics, vol. 102, pp. 69-75, 2014.

[11] D. R. Islamov, V. A. Gritsenko, C. H. Cheng, and A. Chin, "Origin of traps and charge transport mechanism in hafnia," Applied Physics Letters, vol. 105, no. 22, Article ID 222901, 2014.

[12] N. Capron, P. Broqvist, and A. Pasquarello, "Migration of oxygen vacancy in $\mathrm{HfO}_{2}$ and across the $\mathrm{HfO}_{2} / \mathrm{SiO}_{2}$ interface: a first-principles investigation," Applied Physics Letters, vol. 91, no. 19, Article ID 192905, 2007.

[13] L. V. Goncharova, M. Dalponte, D. G. Starodub et al., "Oxygen diffusion and reactions in Hf-based dielectrics," Applied Physics Letters, vol. 89, no. 4, Article ID 044108, 2006.

[14] C. Tang and R. Ramprasad, "Point defect chemistry in amorphous $\mathrm{HfO}_{2}$ : Density functional theory calculations," Physical Review B - Condensed Matter and Materials Physics, vol. 81, no. 16, Article ID 161201, 2010.

[15] K. McKenna and A. Shluger, "The interaction of oxygen vacancies with grain boundaries in monoclinic $\mathrm{HfO}_{2}$," Applied Physics Letters, vol. 95, no. 22, Article ID 222111, 2009.

[16] O. Pirrotta, L. Larcher, M. Lanza et al., "Leakage current through the poly-crystalline $\mathrm{HfO}_{2}$ : trap densities at grains and grain boundaries," Journal of Applied Physics, vol. 114, no. 13, Article ID 134503, 2013.

[17] K. McKenna, A. Shluger, V. Iglesias et al., "Grain boundary mediated leakage current in polycrystalline $\mathrm{HfO}_{2}$ films," Microelectronic Engineering, vol. 88, no. 7, pp. 1272-1275, 2011.

[18] H. Bouwmeester and P. Gellings, Handbook of Solid State Electrochemistry, CRC Press, 1997.

[19] G. Kresse and J. Hafner, "Ab initio molecular dynamics for liquid metals," Physical Review B, vol. 47, no. 1, pp. 558-561, 1993.

[20] G. Kresse and J. Furthmüller, "Efficient iterative schemes for $a b$ initio total-energy calculations using a plane-wave basis set," Physical Review B-Condensed Matter and Materials Physics, vol. 54, no. 16, pp. 11169-11186, 1996.

[21] J. P. Perdew and Y. Wang, "Accurate and simple analytic representation of the electron-gas correlation energy," Physical Review B, vol. 45, no. 23, pp. 13244-13249, 1992.

[22] H. J. Monkhorst and J. D. Pack, "Special points for Brillouinzone integrations," Physical Review. B. Solid State, vol. 13, no. 12, pp. 5188-5192, 1976.

[23] G. K. H. Madsen and D. J. Singh, "BoltzTraP. A code for calculating band-structure dependent quantities," Computer Physics Communications, vol. 175, no. 1, pp. 67-71, 2006.

[24] A. S. Foster, F. Lopez Gejo, A. L. Shluger, and R. M. Nieminen, "Vacancy and interstitial defects in hafnia," Physical Review B Condensed Matter and Materials Physics, vol. 65, no. 17, Article ID 174117, pp. 1741171-17411713, 2002.

[25] K.-H. Xue, P. Blaise, L. R. C. Fonseca et al., "Grain boundary composition and conduction in $\mathrm{HfO}_{2}$ : an ab initio study," Applied Physics Letters, vol. 102, no. 20, Article ID 201908, 2013.

[26] G.-M. Rignanese, "Dielectric properties of crystalline and amorphous transition metal oxides and silicates as potential high- $\kappa$ candidates: The contribution of density-functional theory," Journal of Physics Condensed Matter, vol. 17, no. 7, pp. R357R379, 2005.

[27] J. Wang, H. P. Li, and R. Stevens, "Hafnia and hafnia-toughened ceramics," Journal of Materials Science, vol. 27, no. 20, pp. 53975430, 1992.
[28] H. Jiang, R. I. Gomez-Abal, P. Rinke, and M. Scheffler, "Electronic band structure of zirconia and hafnia polymorphs from the GW perspective," Physical Review B - Condensed Matter and Materials Physics, vol. 81, no. 8, Article ID 085119, 2010.

[29] T. Sohier, M. Calandra, C.-H. Park, N. Bonini, N. Marzari, and F. Mauri, "Phonon-limited resistivity of graphene by firstprinciples calculations: Electron-phonon interactions, straininduced gauge field, and Boltzmann equation," Physical Review $B$ - Condensed Matter and Materials Physics, vol. 90, no. 12, Article ID 125414, 2014.

[30] A. Aziz, P. Mangelis, P. Vaqueiro, A. V. Powell, and R. GrauCrespo, "Electron and phonon transport in shandite-structured $\mathrm{Ni}_{3} \mathrm{Sn}_{2} \mathrm{~S}_{2}$," Physical Review B - Condensed Matter and Materials Physics, vol. 94, no. 16, Article ID 165131, 2016.

[31] M. Fiorentini and N. Bonini, "Thermoelectric coefficients of $\mathrm{n}$-doped silicon from first principles via the solution of the Boltzmann transport equation," Physical Review B - Condensed Matter and Materials Physics, vol. 94, no. 8, Article ID 085204, 2016.

[32] M. J. Wolf, K. P. McKenna, and A. L. Shluger, "Hole trapping at surfaces of $\mathrm{m}-\mathrm{ZrO}_{2}$ and $\mathrm{m}-\mathrm{HfO}_{2}$ nanocrystals," Journal of Physical Chemistry C, vol. 116, no. 49, pp. 25888-25897, 2012.

[33] T. J. Scheidemantel, C. Ambrosch-Draxl, T. Thonhauser, J. V. Badding, and J. O. Sofo, "Transport coefficients from firstprinciples calculations," Physical Review B, vol. 68, no. 12, 2003.

[34] G. K. H. Madsen, "Automated search for new thermoelectric materials: The case of LiZnSb," Journal of the American Chemical Society, vol. 128, no. 37, pp. 12140-12146, 2006.

[35] P. B. Allen, W. E. Pickett, and H. Krakauer, "Anisotropic normalstate transport properties predicted and analyzed for high-Tc oxide superconductors," Physical Review B, vol. 37, no. 13, pp. 7482-7490, 1988. 

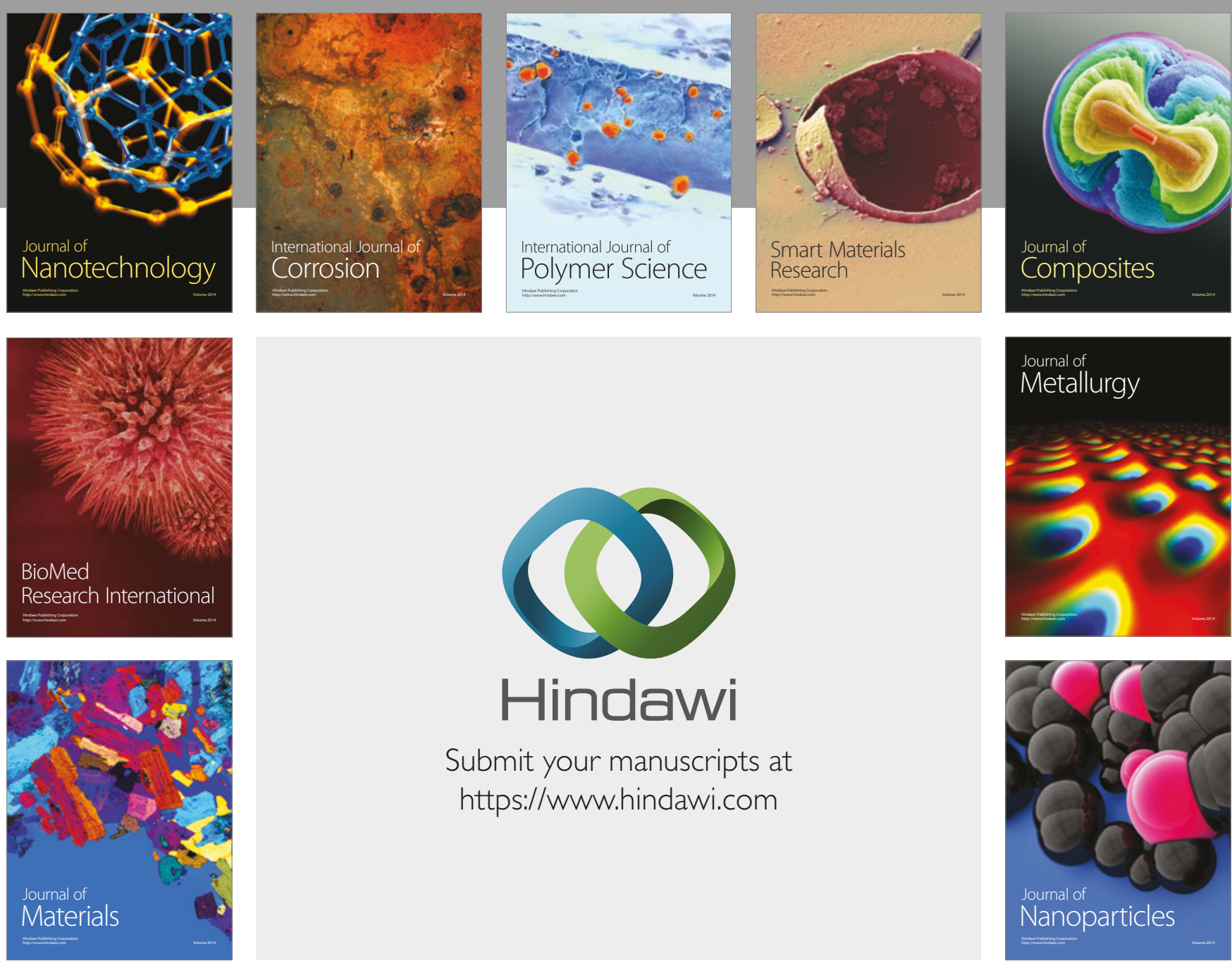

\section{Hindawi}

Submit your manuscripts at

https://www.hindawi.com
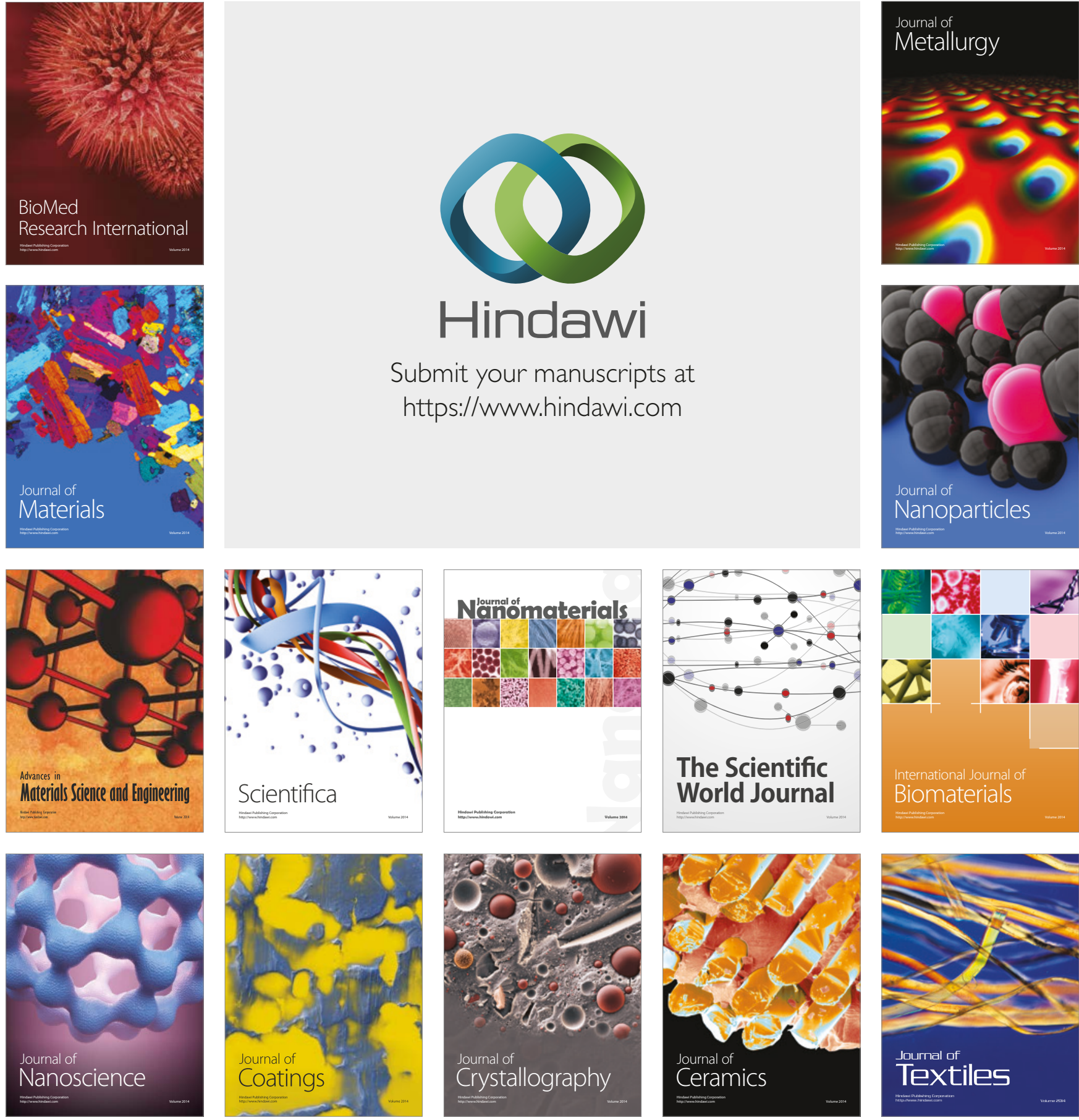

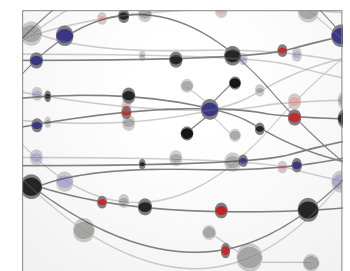

The Scientific World Journal
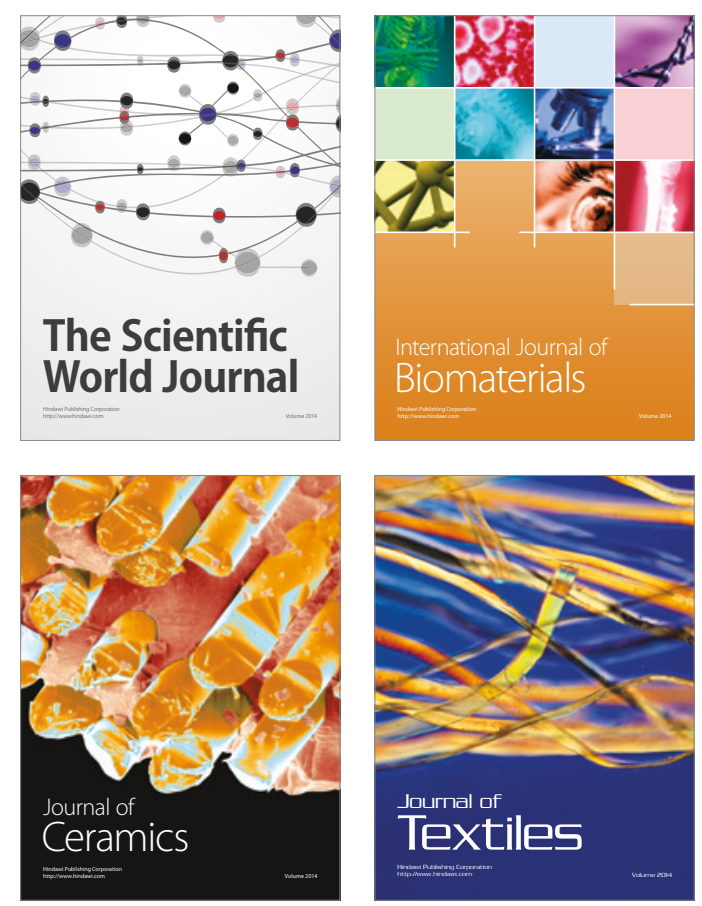